\title{
General Properties of Polymer Systems
}

\author{
C. Gruber and H. KunZ* \\ Laboratoire de Physique Théorique, Ecole Polytechnique Fédérale, Lausanne, Switzerland
}

Received February 19, 1971

\begin{abstract}
We prove the existence of the thermodynamic limit for the pressure and show that the limit is a convex, continuous function of the chemical potential.

The existence and analyticity properties of the thermodynamic limit for the correlation functions is then derived; we discuss in particular the Mayer Series and the virial expansion.

In the special case of Monomer-Dimer systems it is established that no phase transition is possible; moreover it is shown that the Mayer Series for the density is a series of Stieltjes, which yields upper and lower bounds in terms of Padé approximants.

Finally it is shown that the results obtained for polymer systems can be used to study classical lattice systems.
\end{abstract}

\section{Introduction}

In the last decade the mechanism of phase transitions has been intensively investigated by studying properties of rather simple models. One of those models, the Polymer Model [1] (also called quasi-crystalline model), consists of a lattice which is fully covered with non-overlapping "monomers" (molecule occupying one site of the lattice) and "polymers" (rigid system of molecules which can be placed on the lattice in such a way that each molecule of the polymer coincide with one site of the lattice).

This model appears for example in the study of adsorption of polyatomic molecules on a surface; in this case the monomers represent the empty sites. This same model describes physical systems consisting of molecules with unpaired electrons; these molecules then interact to form long chains or polymers. Finally we shall recall that this is also the model which is introduced in the study of liquids consisting of molecules of different sizes or in the so-called cell-cluster theory of liquid state [2].

On the other hand this model is very general since any classical lattice systems (as defined by Ruelle [3]) can be reduced to a system of polymers on the same lattice; this is the "association problem". In some special cases the polymer system associated with the classical lattice

$\star$ Work presented in partial fullfilment of the Ph. D. Thesis. 
system can be further simplified if one consider polymers on a lattice with different geometry [4].

In the present article we study the properties of the polymer model and we prove the existence of the thermodynamic limit for the pressure as well as analyticity properties for the correlation functions at sufficiently high density of monomers. This is obtained using the same formalism and techniques as introduced in the case of classical lattice systems.

Let us mention finally that the more general polymer model in which the molecules which constitute the polymer can be in several possible states can also be reduced to the simple polymer model described above.

\section{Polymer Systems}

\subsection{Definitions}

In this section we describe the model and give the definitions which we shall use in this article.

We consider a finite set $\Lambda$ consisting of $N(\Lambda)$ points in $\mathbb{R}^{v}$ called sites, which are denoted by small letters $x, y, \ldots$ With $X=\left\{x_{1}, \ldots, x_{n}\right\}$ a finite subset of $\Lambda$, a "Polymer $X$ " is a rigid system of $n$ particles which can be placed on $\Lambda$ in such a way as to cover $X$. The polymers consisting of one particle will be called "monomers", of two particles "dimers", ... of $n$ particles " $n$-mers". Polymers are placed on $\Lambda$ and we assume that each site is covered by one and only one particle.

A configuration of the polymer system is therefore defined as a partition $\left\{X_{1}, \ldots, X_{k}\right\}$ of the set $\Lambda$; we recall that by definition of a partition, we have:

$$
\Lambda=\bigcup_{i=1}^{k} X_{i} \quad X_{i} \neq \emptyset \quad X_{i} \cap X_{j}=\emptyset \quad \text { if } \quad i \neq j .
$$

The state of the system is defined as usual by a probability measure $P_{\Lambda}\left(\left\{X_{1}, \ldots, X_{k}\right\}\right)$ on the configuration space; for polymer systems this measure is caracterised by a positive, bounded function $\Phi(X)$ defined on subsets $X \subset \Lambda$, which is interpreted as the "activity of the Polymer $X$ ", and

where

$$
P_{\Lambda}\left(\left\{X_{1}, \ldots, X_{k}\right\}\right)=Q_{\Lambda}^{-1} \prod_{i=1}^{k} \Phi\left(X_{i}\right)
$$

$$
Q_{\Lambda}=\sum_{\substack{\text { all partitions } \\ \Lambda=\cup X_{i}}} \prod_{i} \Phi\left(X_{i}\right) \quad \text { is the "Partition Function". }
$$

The pressure $p_{A}$ is defined by:

$$
\beta p_{\Lambda}=\frac{1}{N(\Lambda)} \log Q_{\Lambda} \quad \text { where } \beta=\frac{1}{k T} .
$$


We shall also study the correlation functions $\varrho_{A}\left(X_{1} ; \ldots ; X_{p}\right)$ which are defined as the probability of finding polymers $X_{1}, \ldots, X_{p}$. With the above probability measure we have:

$$
\begin{array}{r}
\varrho_{\Lambda}\left(X_{1} ; \ldots ; X_{p}\right)=0 \quad \text { if } X_{i} \cap X_{j} \neq \emptyset \text { for some } i \neq j \\
\varrho_{\Lambda}\left(X_{1} ; \ldots ; X_{p}\right)=Q_{\Lambda}^{-1} \prod_{i=1}^{p} \Phi\left(X_{i}\right)\left\{\sum_{\substack{\text { all partitions } \\
\Lambda \backslash \cup X_{i}=\cup Y_{j}}} \Pi \Phi\left(Y_{j}\right)\right\} \\
\text { if } X_{i} \cap X_{j}=\emptyset \text { for all } i \neq j .
\end{array}
$$

With these definitions the correlation functions have in particular the following properties

i) $0 \leqq \varrho_{\Lambda}\left(X_{1} ; \ldots ; X_{p}\right) \leqq 1$,

ii) $\varrho_{\Lambda}(X)=\Phi(X) \frac{\partial}{\partial \Phi(X)} \log Q_{\Lambda}=\frac{1}{\beta} \frac{\partial}{\partial \mu(X)} \log Q_{\Lambda}$

where $\beta \mu(X)=\log \Phi(X)$

and the density of " $n$-mers" $\varrho_{A}^{(n)}$ is defined by

$$
\varrho_{\Lambda}^{(n)}=\frac{1}{N(\Lambda)} \sum_{\substack{X \subset A \\ N(X)=n}} \varrho_{\Lambda}(X)=\sum_{\substack{X \subset A \\ N(X)=n}} \frac{\partial p_{\Lambda}}{\partial \mu(X)}
$$

where the sum is restricted to those subsets containing $N(X)=n$ elements.

Finally if $\Lambda$ is a subset of $\mathbb{Z}^{v}$, we shall say that the activity is translationally invariant if it satisfies

and

$$
\Phi\left(\tau_{a} X\right)=\Phi(X) \text { for all } a \in \mathbb{Z}^{v} \text { and } X \subset \mathbb{Z}^{v}
$$

$$
\tau_{a} X=\left\{x_{1}+a, \ldots, x_{n}+a\right\} \text { for all } X=\left\{x_{1}, \ldots, x_{n}\right\} .
$$

\subsection{Notation and Algebraic Formalism}

In order to obtain a concise formulation of the problem, we shall make use of an algebraic formalism which has already been used extensively in statistical mechanics [5].

Let $X=\left\{x_{1}, \ldots, x_{p}\right\}$ and $Y=\left\{y_{1}, \ldots, y_{q}\right\}$ denote subsets of $\Lambda$.

If $X \cap Y=\emptyset$ we shall write $X \cup Y$ as $X+Y$.

If $Y \subset X$ we shall write $X \backslash Y$ as $X-Y$.

We shall denote by $N(X)$ the number of elements in the subset $X$; finally $\sum_{X=\Sigma X_{l}}$ will mean "sum over all partitions of $X$ ". 
Let $V_{A}$ be the complex vector space of bounded functions $\Psi(X)$ defined on the subsets $X \subset \Lambda$; this vector space is a commutative algebra with unit element $\mathbb{1}$ for the *-product defined by:

$$
\forall \Psi_{1}, \Psi_{2} \in V_{A}, \quad\left(\Psi_{1} * \Psi_{2}\right)(X)=\sum_{Y \subset X} \Psi_{1}(Y) \Psi_{2}(X-Y) .
$$

The unit element $\mathbb{1}$ is the vector defined by:

$$
\mathbb{1}(X)=\left\{\begin{array}{lll}
1 & \text { if } & X=\emptyset \\
0 & \text { if } & X \neq \emptyset .
\end{array}\right.
$$

We also define a mapping $\Gamma$ of $V_{A}^{+}=\left\{\Psi \mid \Psi \in V_{A} \Psi(\emptyset)=0\right\}$ on $\mathbb{1}+V_{A}^{+}$ by means of the $*$-exponential:

which gives explicitely:

$$
\Gamma \Psi=\mathbb{1}+\sum_{n=1}^{\infty} \frac{1}{n !} \Psi^{* n}
$$

$$
(\Gamma \Psi)(X)=\sum_{X=\Sigma X_{i}} \prod_{i} \Psi\left(X_{i}\right) .
$$

Finally we define an operation $D_{X}: \Psi \rightarrow D_{X} \Psi$

where

$$
\left(D_{X} \Psi\right)(Y)=\Psi(X+Y) \delta_{\emptyset, X \cap Y}
$$

$$
\delta_{\emptyset, T}=\left\{\begin{array}{lll}
1 & \text { if } & \emptyset=T \\
0 & \text { if } & \emptyset \neq T .
\end{array}\right.
$$

This operation satisfies in particular the following relations:

$$
\begin{aligned}
D_{x}\left(\Psi_{1} * \Psi_{2}\right)(Y) & =\left[\left(D_{x} \Psi_{1} * \Psi_{2}\right)(Y)+\left(\Psi_{1} * D_{x} \Psi_{2}\right)(Y)\right] \delta_{\emptyset,\{x\} \cap Y} \\
\left(D_{x} \Gamma \Psi\right)(Y) & =\left(D_{x} \Psi * \Gamma \Psi\right)(Y) \delta_{\emptyset,\{x\} \cap Y} \\
\left(D_{X} \Gamma \Psi\right)(Y) & =\sum_{\substack{q \\
X=\sum_{1} X_{i}}}\left(D_{X_{1}} \Psi * \cdots * D_{X_{q}} \Psi * \Gamma \Psi\right)(Y) \delta_{\emptyset, X \cap Y} .
\end{aligned}
$$

There is one more property of the mapping $\Gamma$ which we shall need later:

Defining

we have

$$
\lambda^{N} \Psi \quad \text { by } \quad\left(\lambda^{N} \Psi\right)(X)=\lambda^{N(X)} \Psi(X) \quad \forall \lambda \in \mathbb{C}
$$

$$
\Gamma\left(\lambda^{N} \Psi\right)=\lambda^{N} \Gamma \Psi
$$

\subsection{Algebraic Formulation of the Polymer System and Scaling Property}

Taking $\Phi(\emptyset)=0$, the algebraic formalism just introduced, enables us to write the partition function and the correlation functions in the 
following form:

$$
\begin{gathered}
Q_{\Lambda}=(\Gamma \Phi)(\Lambda), \\
\varrho_{\Lambda}\left(X_{1} ; \ldots ; X_{p}\right)=\prod_{i=1}^{p} \Phi\left(X_{i}\right) \frac{(\Gamma \Phi)\left(\Lambda-\sum_{i=1}^{p} X_{i}\right)}{(\Gamma \Phi)(\Lambda)} \text { if } X_{i} \cap X_{j}=\emptyset .
\end{gathered}
$$

This expression for the correlation functions suggests to introduce the reduced correlation functions $\bar{\varrho}_{\Lambda}(X)$ defined as:

which gives:

$$
\bar{\varrho}_{\Lambda}(X)=\frac{(\Gamma \Phi)(\Lambda-X)}{(\Gamma \Phi)(\Lambda)}
$$

$$
\varrho_{\Lambda}\left(X_{1} ; \ldots ; X_{p}\right)=\prod_{i=1}^{p} \Phi\left(X_{i}\right) \quad \bar{\varrho}_{\Lambda}\left(\sum_{i} X_{i}\right)
$$

Using this formalism we obtain at once the following sum rules:

and

$$
\sum_{X \ni x} \varrho_{\Lambda}(X)=1
$$

$$
\sum_{n=1}^{\infty} n \varrho_{\Lambda}^{(n)}=\frac{1}{N(\Lambda)} \sum_{X \subset \Lambda} N(X) \varrho_{\Lambda}(X)=1
$$

Indeed Eq. (14) yields:

$$
Q_{\Lambda}=(\Gamma \Phi)(\Lambda)=\left(D_{x} \Gamma \Phi\right)(\Lambda-x)=\sum_{X \ni x} \Phi(X)(\Gamma \Phi)(\Lambda-X)
$$

dividing both sides by $Q_{\Lambda}$ gives Eq. (20).

Eq. (21) follows from:

$$
\sum_{X \subset \Lambda} N(X) \varrho_{\Lambda}(X)=\sum_{x \in \Lambda} \sum_{X \ni x} \varrho_{\Lambda}(X)=N(\Lambda) .
$$

We notice that these sum rules express only the fact that each site is occupied by one and only one particle.

In the cases where $\Lambda$ is taken to be a finite subset of $\mathbb{Z}^{v}, \bar{\varrho}_{\Lambda}$ will be defined for all $X \subset \mathbb{Z}^{v}$ by:

$$
\bar{\varrho}_{\Lambda}(X)=\chi_{\Lambda}(X) \frac{(\Gamma \Phi)(\Lambda-X)}{(\Gamma \Phi)(\Lambda)}
$$

where $\chi_{A}$ is the caracteristic function of the set of subsets of $\Lambda$ (in the set of subsets of $\mathbb{Z}^{v}$ ).

We shall write $Q_{A}[\Phi]$ and $\varrho_{A}[\Phi]$ when we want to investigate the dependence of $Q_{\Lambda}$ and $\varrho_{\Lambda}$ on the activity $\Phi$. From the property (15) of the mapping $\Gamma$, we deduce immediately the following scaling properties:

10 Commun math. Phys., Vol. 22 
For all $\lambda \in \mathbb{C}$

therefore

$$
Q_{\Lambda}[\Phi]=\lambda^{-N(\Lambda)} Q_{\Lambda}\left[\lambda^{N} \Phi\right]
$$

$$
\begin{aligned}
\beta p_{\Lambda}[\Phi] & =\beta p_{\Lambda}\left[\lambda^{N} \Phi\right]-\log \lambda \\
\varrho_{\Lambda}[\Phi] & =\varrho_{\Lambda}\left[\lambda^{N} \Phi\right] \\
\bar{\varrho}_{\Lambda}[\Phi] & =\lambda^{N} \bar{\varrho}_{\Lambda}\left[\lambda^{N} \Phi\right] .
\end{aligned}
$$

This scaling property implies in particular the following:

Proposition. Consider a system made up only of monomers and $\alpha$-mers ( $n$ fixed; $\alpha=1,2, \ldots$ ) and such that the activity $\Phi(x)$ of the monomers is constant $\Phi(x)=z$.

i) The set of zeros of the partition function $Q_{A}$ as function of the complex variable $z$ possess the symmetry $C_{n}$ with respect to the origin $\left(C_{n}=\right.$ cyclic group of order $\left.n\right)$.

ii) The domain of analyticity of the correlation function in the complex variable z possess the symmetry $C_{n}$.

\section{Thermodynamic Limit for the Pressure}

To study the existence and properties of this thermodynamic limit, we shall consider $\Lambda$ to be a finite subset of $\mathbb{Z}^{v}$ and we shall assume the activity to be translationally invariant.

The activity will be said to be stable if it satisfies the following condition:

$$
\|\Phi\|=\sum_{X \ni 0} \frac{|\Phi(X)|}{N(X)}<\infty
$$

\subsection{Existence of the Limit}

Lemma 1. If the activity $\Phi$ is stable and non-negative $\left(\Phi(X) \geqq 0 \forall X \subset \mathbb{Z}^{v}\right)$ then

i) $\log z \leqq \beta p_{\Lambda} \leqq\|\Phi\|$

where $z=\Phi(x)$ is the activity of monomers.

ii) for $z>0$, the function $-N(\Lambda) p_{\Lambda}$ is subadditive with respect to $\Lambda$.

Proof. i) The lower bound is a direct consequence of the scaling property (22):

$$
z^{-N(\Lambda)} Q_{\Lambda}[\Phi]=Q_{\Lambda}\left[z^{-N} \Phi\right]=1+\sum_{p=1}^{N(\Lambda)-1} \sum_{\substack{p \\ \Lambda=1}} z^{-N(\Lambda)} \prod_{i=1}^{p} \Phi\left(X_{i}\right) \geqq 1
$$

therefore $-\log z+\beta p_{\Lambda} \geqq 0$. 
The upper bound follows from the stability condition since:

therefore

$$
\begin{aligned}
Q_{\Lambda}[\Phi]= & (\Gamma \Phi)(\Lambda)=\sum_{\Lambda=\Sigma X_{i}} \prod_{i} \Phi\left(X_{i}\right)=\sum_{\Lambda=\Sigma X_{i}} \prod_{i} \sum_{x \in X_{i}} \frac{\Phi\left(X_{i}\right)}{N\left(X_{i}\right)} \\
& \leqq \sum_{p} \frac{1}{p !} \prod_{i=1}^{p} \sum_{x \in \Lambda} \sum_{X_{i} \ni x} \frac{\Phi\left(X_{i}\right)}{N\left(X_{i}\right)} \leqq \exp N(\Lambda)\|\Phi\|
\end{aligned}
$$

$$
\beta p_{\Lambda}=\frac{1}{N(\Lambda)} \log Q_{\Lambda} \leqq\|\Phi\|
$$

ii) $Q_{\Lambda_{1}+\Lambda_{2}}=(\Gamma \Phi)\left(\Lambda_{1}+\Lambda_{2}\right)=\left(D_{\Lambda_{1}} \Gamma \Phi\right)\left(\Lambda_{2}\right)$

$$
=(\Gamma \Phi)\left(\Lambda_{1}\right)(\Gamma \Phi)\left(\Lambda_{2}\right)+\sum_{\Lambda_{1}=\Sigma X_{j}} \sum_{\substack{S \subset \Lambda_{2} \\ S \neq \emptyset}}\left(D_{X_{1}} \Phi * \cdots * D_{X_{q}} \Phi\right)(S)(\Gamma \Phi)\left(\Lambda_{2}-S\right)
$$

therefore

and

$$
Q_{\Lambda_{1}+\Lambda_{2}} \geqq Q_{\Lambda_{1}} Q_{\Lambda_{2}}
$$

$$
-N\left(\Lambda_{1}+\Lambda_{2}\right) p_{\Lambda_{1}+\Lambda_{2}} \leqq-N\left(\Lambda_{1}\right) p_{\Lambda_{1}}-N\left(\Lambda_{2}\right) p_{\Lambda_{2}} .
$$

Theorem 1. For any non-negative, stable activity $\Phi(X)$ such that $\Phi(x)=z \neq 0$, the following limit exists and is finite

$$
\begin{gathered}
\beta p=\lim _{\Lambda \rightarrow \infty} \beta p_{\Lambda} \\
\log z \leqq \beta p \leqq\|\Phi\|
\end{gathered}
$$

when $\Lambda$ tends to $\infty$ in the following sense [6]

a) $\Lambda \rightarrow \infty$ in the sense of Van Hove,

b) there exists $C>0$ and for each $\Lambda$, a parallelipiped $\Lambda(b)$ with sides $\left(b_{1}, \ldots, b_{v}\right)$ such that $\Lambda \subset \Lambda(b), N(\Lambda(b))^{-1} N(\Lambda)>C>0$ and $b \rightarrow \infty$.

Proof. The subadditivity of $-N(\Lambda) p_{\Lambda}$ and the fact that $-\beta p_{A} \geqq-\|\Phi\|$ implies that the following limit exists

$$
\lim _{a \rightarrow \infty} p_{\Lambda(a)}=\sup _{a} p_{\Lambda(a)}=p
$$

where $a \rightarrow \infty$ means that each side of the parallelipiped $\Lambda(a)$ tends to $\infty$.

To show the existence of the limit in the more general sense we consider a sequence of volume $\Lambda_{j}$ which tends to $\infty$ in the sense of our theorem.

1) By definition of Van Hove Convergence, for all $a$ there exists unions of parallelepipeds with sides $a$, say $\Lambda_{j}^{-}, \Lambda_{j}^{+}$such that:

$$
\Lambda_{j}^{-} \subset \Lambda_{j} \subset \Lambda_{j}^{+} \text {and } \frac{N\left(\Lambda_{j}^{ \pm}\right)}{N\left(\Lambda_{j}\right)} \underset{j \rightarrow \infty}{\longrightarrow} 1 \text {. }
$$


Introducing the quantity $f_{A}=\beta p_{A}-\log z$, Lemma 1 implies that $f_{\Lambda}$ is positive and $-N(\Lambda) f_{\Lambda}$ is subadditive; from this follows that

$$
f_{\Lambda_{j}} \geqq \frac{N\left(\Lambda_{j}^{-}\right)}{N\left(\Lambda_{j}\right)} f_{\Lambda(a)}
$$

therefore $\lim \inf f_{A_{j}} \geqq \sup _{a} f_{\Lambda(a)}=f=\beta p-\log z$.

2) Let $\Lambda(b)$ be a parallelipiped made up of parallelipipeds $\Lambda(a)$ and such that $\Lambda(b) \supset \Lambda_{j}^{+} \supset \Lambda_{j}$

$$
\begin{gathered}
N(\Lambda(b)) f_{\Lambda(b)} \geqq N\left(\Lambda_{j}\right) f_{\Lambda_{j}}+\left[N\left(\Lambda_{b}\right)-N\left(\Lambda_{j}^{+}\right)\right] f_{\Lambda(a)}, \\
f_{\Lambda_{j}} \leqq \frac{N(\Lambda(b))}{N\left(\Lambda_{j}\right)} f-\frac{N\left(\Lambda_{b}\right)-N\left(\Lambda_{j}^{+}\right)}{N\left(\Lambda_{j}\right)} f_{\Lambda(a)} .
\end{gathered}
$$

Since $f=\sup _{a} f_{\Lambda(a)}$, for all $\varepsilon>0$ there exists $a$ such that $f_{\Lambda(a)}>f-\varepsilon$; therefore

$$
f_{\Lambda_{j}} \leqq \frac{N\left(\Lambda_{j}^{+}\right)}{N\left(\Lambda_{j}\right)} f+\frac{N\left(\Lambda^{(b)}\right)}{N\left(\Lambda_{j}\right)} \varepsilon .
$$

Condition (a) and (b) implies then

$$
\lim \sup f_{\Lambda_{j}} \leqq f .
$$

Finally using the scaling property (22) we obtain:

Corollary. Theorem 1 remains valid for any activity of the form $\xi^{N(X)} \Phi(X)$ where $\xi$ is a positive number and $\Phi(X)$ satisfies the conditions of Theorem 1.

\subsection{Convexity and Continuity Property ${ }^{1}$}

In this section we shall investigate the convexity and continuity properties of the functions $p_{A}$ and $p$; these properties are obtained in terms of the chemical potential $\mu(X)$ introduced in Section 2 as

$$
\beta \mu(X)=\log \Phi(X) .
$$

The condition $\Phi(X)$ is stable and non-negative is equivalent to the condition $\mu(X) \in \mathscr{C}$ where

$$
\mathscr{C}=\left\{\mu(X) \mid \mu(X) \in \mathbb{R} \quad X \subset \mathbb{Z}^{v} \quad \text { and } \quad \sum_{X \ni 0} \frac{e^{\beta \mu(X)}}{N(X)}<\infty\right\}
$$

Since we shall consider $p_{A}$ as a functional of the chemical potential $\mu(X)$ we shall write:

$$
p_{\Lambda}[\Phi]=P_{\Lambda}[\mu] \text { where } \Phi(X)=e^{\beta \mu(X)} .
$$

${ }^{1}$ We recall that we have assumed in this chapter that $\Phi$ and $\mu$ are translationally invariant. 
Finally we introduce the Banach Space $\mathscr{B}$ of real chemical potential $v(X)$ satisfying:

$$
\|v\| \|=\sup _{X \subset \mathbb{Z}^{v}}|v(X)|<\infty .
$$

Lemma 2. i) The function $\mu \rightarrow P_{\Lambda}[\mu]$ is convex on $\mathscr{C}$.

ii) For all $\mu \in \mathscr{C}$ and $v \in \mathscr{B}$

$$
\left|P_{\Lambda}[\mu+v]-P_{\Lambda}[\mu]\right| \leqq\|v\| .
$$

Hence for fixed $\mu \in \mathscr{C}$ the function $v \rightarrow P_{\Lambda}[\mu+v]$ is continuous on the Banach Space $\mathscr{B}$.

Proof. i) The convexity of $\mathscr{C}$ follows from the convexity of the exponential; the convexity of $P_{A}$ from Hölder inequality.

$$
\begin{gathered}
Q_{\Lambda}[\alpha \mu+(1-\alpha) v]=\sum_{\Lambda=\Sigma X_{i}} e^{\beta \alpha \Sigma \mu\left(X_{i}\right)+\beta(1-\alpha) \Sigma v\left(X_{i}\right)} \\
\leqq\left[\sum_{\Lambda=\Sigma X_{i}} e^{\beta \Sigma \mu\left(X_{i}\right)}\right]^{\alpha}\left[\sum_{\Lambda=\Sigma X_{i}} e^{\beta \Sigma v\left(X_{i}\right)}\right]^{1-\alpha}, \\
Q_{\Lambda}[\alpha \mu+(1-\alpha) v] \leqq Q_{\Lambda}[\mu]^{\alpha} Q_{\Lambda}[v]^{1-\alpha} .
\end{gathered}
$$

ii) We remark first that for all $\mu \in \mathscr{C}$ and $v \in \mathscr{B}, \mu+v \in \mathscr{C}$ since:

Moreover:

$$
\sum_{X \ni 0} \frac{e^{\beta(\mu(X)+v(X))}}{N(X)} \leqq e^{\beta\|v v\| \mid} \sum_{X \ni 0} \frac{e^{\beta \mu(X)}}{N(X)}<\infty .
$$

implies

$$
Q_{\Lambda}[\mu+v]=\sum_{\Lambda=\Sigma X_{i}} e^{\beta \Sigma\left(\mu\left(X_{t}\right)+v\left(X_{i}\right)\right)}
$$

$$
\begin{gathered}
e^{-\beta N(A)\|v\|} Q_{\Lambda}[\mu] \leqq Q_{\Lambda}[\mu+v] \leqq e^{\beta N(\Lambda)\|v\|} Q_{\Lambda}[\mu] \\
-\|v\|+P_{\Lambda}[\mu] \leqq P_{\Lambda}[\mu+v] \leqq\|v\|+P_{\Lambda}[\mu] .
\end{gathered}
$$

Theorem 2. With

$$
P[\mu]=\lim _{\Lambda \rightarrow \infty} P_{\Lambda}[\mu] .
$$

i) The function $\mu \rightarrow P[\mu]$ is convex on $\mathscr{C}$.

ii) For fixed $\mu \in \mathscr{C}$, the function $v \rightarrow P[\mu+v]$ is continuous on the Banach space $\mathscr{B}$.

This theorem is a direct consequence of Theorem 1 and Lemma 2.

\subsection{Thermodynamic Limit for the Tangent Plane to the Graph of $P$}

To conclude this paragraph we shall derive a result concerning the existence of the thermodynamic limit for the tangent plane to the graph of $P$. Following Ruelle [7] we introduce the average correlation func- 
tion $\tilde{\varrho}_{\Lambda}(X)$ :

$$
\tilde{\varrho}_{\Lambda}(X)=\frac{1}{N(\Lambda)} \sum_{a \in \mathbb{Z}^{\nu}} \varrho_{\Lambda}\left(\tau_{a} X\right)
$$

which represents the density of polymer of type $X$ for the system $A$ (i.e. the density of polymers having $N(X)$ elements and the same oriented shape as $X$ ).

Theorem 3. For fixed $\mu \in \mathscr{C}$, we consider the function $v \rightarrow P[\mu+v]$ defined on the Banach space $\mathscr{B}$. If the chemical potential $\mu(X)$ is such that $v \rightarrow P[\mu+v]$ has a unique tangent plane $\alpha^{\mu}[v]$ at the point $v=0$, then when $\Lambda \rightarrow \infty$ in the sense of Theorem 1, we have:

i) $\lim _{\Lambda \rightarrow \infty} \sum_{X \ni 0} \tilde{\varrho}_{\Lambda}^{\mu}(X) \frac{v(X)}{N(X)}=-\alpha^{\mu}[v]$ for all $v \in \mathscr{B}$,

ii) for every finite $X \subset \mathbb{Z}^{v}$ the following limit exists

$$
\lim _{\Lambda \rightarrow \infty} \tilde{\varrho}_{\Lambda}^{\mu}(X)=\tilde{\varrho}^{\mu}(X)
$$

and defines the infinite volume density of polymer of type $X$,

iii) the following limit exists

$$
\lim _{\Lambda \rightarrow \infty} \varrho_{\Lambda}^{(n)}=\varrho^{(n)}=\sum_{\substack{X \subset \mathbb{Z}^{\nu} \\ N(X)=n}} \frac{\partial p}{\partial v(X)}
$$

and defines the density of $n$-mers for infinite systems (where $\varrho_{\Lambda}^{(n)}$ is defined by Eq. (8)).

Proof. i) For any finite $\Lambda$ and any $\mu \in \mathscr{C}$, the function $v \rightarrow P_{\Lambda}[\mu+v]$ has a unique tangent plane at $v=0$; indeed

$$
\begin{aligned}
\left.\frac{d P_{\Lambda}[\mu+\lambda v]}{d \lambda}\right|_{\lambda=0} & =-\alpha_{\Lambda}^{\mu}[v]=\frac{1}{Q_{\Lambda}} \frac{1}{N(\Lambda)} \sum_{\Lambda=\Sigma X_{i}} e^{\beta \Sigma \mu\left(X_{i}\right)}\left(\Sigma v\left(X_{i}\right)\right) \\
& =\frac{1}{Q_{\Lambda}} \frac{1}{N(\Lambda)} \sum_{X \subset \Lambda} v(X) \sum_{\Lambda-X=\Sigma Y_{j}} e^{\beta \mu(X)} e^{\beta \Sigma \mu\left(Y_{j}\right)} \\
& =\frac{1}{N(\Lambda)} \sum_{X \subset \Lambda} v(X) \varrho_{\Lambda}(X)=\sum_{X \ni 0} \tilde{\varrho}_{\Lambda}(X) \frac{v(X)}{N(X)} .
\end{aligned}
$$
Finally Eq. (21) implies that $\sum_{X} \varrho_{\Lambda}(X) \leqq 1$ and therefore for all $v \in \mathscr{B}$,
$\alpha_{\Lambda}^{\mu}[v]$ exists and satisfies

$$
\left|\alpha_{\Lambda}^{\mu}[v]\right| \leqq\|v \mid\| \text {. }
$$

The end of the proof is identical to the one given by Gallavotti and Miracle [8].

ii) This follows immediately from part (i). 
We remark that the density of polymer of type $X$ is up to the factor $-\frac{1}{N(X)}$ the functional derivative of $P_{\Lambda}$ with respect to $v(X)$.

iii) From the definition of $\varrho_{A}^{(n)}$ we have:

$$
\varrho_{\Lambda}^{(n)}=\sum_{\substack{X \\ N(X)=n}} \tilde{\varrho}_{\Lambda}(X) \frac{1}{N(X)}=-\alpha_{\Lambda}^{\mu}\left[v_{n}\right]
$$

where $v_{n}(X)=\delta_{n, N(X)} \in \mathscr{B}$.

To conclude this theorem we remark [8] that if

$$
-\left.\frac{d P(\mu+\lambda v)}{d \lambda}\right|_{\lambda=0}=\alpha^{\mu}[v]
$$

exists for a certain $v \in \mathscr{B}$ then

$$
\lim _{\Lambda \rightarrow \infty} \alpha_{\Lambda}^{\mu}[v]=\alpha^{\mu}[v] .
$$

Remarks. i) The existence of the thermodynamic limit was obtained only for the case $z \neq 0$; for the case $z=0$, it is in fact possible to find two sequences of volume which tends to $\infty$ and such that the pressure converges to different values.

ii) The continuity of the pressure, and the functional derivative, was obtained relative to the chemical potential $v(X)$; this is connected with the fact that systems which do not have their activities $\Phi(X)$ equal to zero on the same subsets $X$ are different physical systems.

iii) The results obtained in this section are in close analogy with those obtained for lattice systems; it is however interesting to notice that in the case of polymers, the functional derivatives do not provide a complete description of the state of the system. (Indeed by functional derivativation one can obtain $\varrho(X)$ only for those $X$ such that $\Phi(X) \neq 0$.)

\section{Thermodynamic Limit for the Correlation Functions}

To study the existence and properties of the correlation functions for infinite systems, we shall first derive a set of equations which are satisfied by the reduced correlation functions of finite systems $\bar{\varrho}_{A}$. From this set of equations, it is possible to extract several minimal subsets of equations equivalent to the full set; in particular one of those minimal subsets is analogous to the Kirkwood-Salsburg equations for classical continuous systems. We shall investigate this particular set of equations using the well-known methods of Banach space and Neumann series.

In this section we shall consider $\Lambda$ to be a subset of $\mathbb{Z}^{v}$; however we do not restrict ourselves to the case of translation invariant activities. 


\subsection{Equations for the Correlation Functions}

From the general properties of the mapping $\Gamma$ introduced in $\S 2$, we obtain:

$$
=(\Gamma \Phi)(Y)(\Gamma \Phi)(Z)+\sum_{Y=\Sigma Y_{\imath}} \sum_{\substack{S \subset Z \\ S \neq \emptyset}}\left(D_{Y_{1}} \Phi * \cdots * D_{Y_{q}} \Phi\right)(S)(\Gamma \Phi)(Z-S) .
$$

If $(\Gamma \Phi)(\Lambda) \neq 0$, writing $Z=\Lambda-X$ and with $Y \subset X \subset \Lambda$ we then have:

$$
\bar{\varrho}_{\Lambda}(X-Y)=(\Gamma \Phi)(Y) \bar{\varrho}_{\Lambda}(X)+\sum_{Y=\Sigma Y_{i}} \sum_{\substack{S \subset A-X \\ S \neq \emptyset}}\left(D_{Y_{1}} \Phi * \cdots * D_{Y_{p}} \Phi\right)(S) \bar{\varrho}_{\Lambda}(X+S) .
$$

Therefore for any finite volume $\Lambda$, and for any activities such that $(\Gamma \Phi)(\Lambda) \neq 0$, the correlation functions satisfy for all $X$ and $Y \subset X$ :

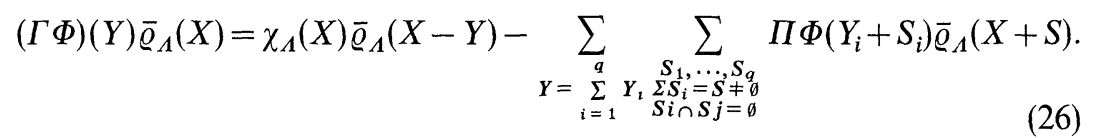

This relation can also be written as $^{2}$ :

$$
(\Gamma \Phi)(Y) \bar{\varrho}_{\Lambda}(X)=\chi_{\Lambda}(X) \bar{\varrho}_{\Lambda}(X-Y)-\sum_{\substack{S \neq \emptyset \\ S \cap X=\emptyset}}\left[(\Gamma \Phi)^{-1} * D_{Y} \Gamma \Phi\right](S) \bar{\varrho}_{\Lambda}(X+S)
$$

At this point we would like to find minimal subsets of (26) that are equivalent to the full set of equations.

Lemma 3. If $\bar{\varrho}_{A}$ satisfies (26) for all $Y \subset X$ such that $N(Y) \leqq n$, then it satisfies (26) for all $Y$ such that $N(Y)=n+1$.

Proof. Let $Y \subset X$ be any subset such that $N(Y)=n$.

From the relations and identities:

$$
(\Gamma \Phi)(Y+\{y\})=\sum_{S \subset Y} \Phi(\{y\}+S)(\Gamma \Phi)(Y-S) \quad y \in X-Y
$$

$$
\begin{aligned}
\sum_{S \subset Y} \Phi(\{y\}+S) \bar{\varrho}_{A}(X-Y+S) \\
=\sum_{S \subset Y} \Phi(\{y\}+S)\left[\bar{\varrho}_{\Lambda}(X-Y+S)+\bar{\varrho}_{\Lambda}(X-Y-\{y\})\right] \\
\quad-\sum_{S \cap(X-Y)=\emptyset} \Phi(\{y\}+S) \bar{\varrho}_{\Lambda}(X-Y+S) \\
=\bar{\varrho}_{\Lambda}(X-Y-\{y\})-\sum_{\substack{Y_{1} \subset Y \\
U_{1} \cap X=\emptyset \\
U_{1} \neq \emptyset}} \Phi\left(\{y\}+Y_{1}+U_{1}\right) \bar{\varrho}_{\Lambda}\left(X+U_{1}-\left(Y-Y_{1}\right)\right)
\end{aligned}
$$

${ }^{2}(\Gamma \Phi)^{-1}$ denotes the $*$-inverse of $\Gamma \Phi$. 
and

$$
\begin{aligned}
\sum_{S \subset Y}(\Gamma \Phi)(U+Y-S) \Phi(\{y\}+S) \\
=\sum_{\substack{S \subset Y+U\\
}}(\Gamma \Phi)(U+Y-S) \Phi(\{y\}+S) \\
\quad-\sum_{\substack{Y_{1} \subset Y \\
U_{1} \subset U \\
U_{1} \neq \emptyset}}(\Gamma \Phi)\left(U-U_{1}+Y-Y_{1}\right) \Phi\left(\{y\}+U_{1}+Y_{1}\right) \\
=\left(D_{\{y\}+Y} \Gamma \Phi\right)(U)-\sum_{\substack{Y_{1} \subset Y \\
U_{1} \subset U \\
U_{1} \neq \emptyset}}(\Gamma \Phi)\left(U-U_{1}+Y-Y_{1}\right) \Phi\left(\{y\}+U+Y_{1}\right)
\end{aligned}
$$

the hypothesis of the lemma implies that for any $y \in X-Y$

$$
\begin{aligned}
(\Gamma \Phi)(\{y\}+Y) \bar{\varrho}_{\Lambda}(X)=\sum_{S \subset Y} \Phi(\{y\}+S)\left[(\Gamma \Phi)(Y-S) \bar{\varrho}_{\Lambda}(X)\right] \\
=\sum_{S \subset Y} \Phi(\{y\}+S)\left[\chi_{\Lambda}(X) \bar{\varrho}_{\Lambda}(X+S-Y)\right. \\
\left.\quad-\sum_{\substack{T \neq \emptyset \\
T \cap X=\emptyset}}\left[(\Gamma \Phi)^{-1} * D_{Y-S} \Gamma \Phi\right](T) \bar{\varrho}_{\Lambda}(X+T)\right] \\
=\chi_{\Lambda}(X) \bar{\varrho}_{\Lambda}(X-Y-\{y\}) \\
\quad-\sum_{\substack{U_{1} \subset X=\emptyset \\
U_{1} \neq \emptyset}} \Phi\left(\{y\}+Y_{1}+U_{1}\right) \chi_{\Lambda}(X) \bar{\varrho}_{\Lambda}\left(X+U_{1}-\left(Y-Y_{1}\right)\right) \\
\quad-\sum_{\substack{T \neq \emptyset \\
T \cap X=\emptyset}}\left\{(\Gamma \Phi)^{-1} * D_{\{y\}+Y} \Gamma \Phi\right](T) \bar{\varrho}_{\Lambda}(X+T) \\
+\sum_{Y_{1} \subset Y} \sum_{U \subset T \sum_{U_{1} \subset U}} \sum_{U_{1} \neq \emptyset} \Phi\left(\{y\}+U_{1}+Y_{1}\right) \\
\left.\quad \cdot \quad(\Gamma \Phi)^{-1}(T-U)(\Gamma \Phi)\left(U-U_{1}+Y-Y_{1}\right) \bar{\varrho}_{\Lambda}(X+T)\right\} .
\end{aligned}
$$

The last term being equal to:

$$
\begin{aligned}
& \sum_{\substack { Y_{1} \subset Y \\
\begin{subarray}{c}{T \neq \emptyset \\
T \cap X=\emptyset{ Y _ { 1 } \subset Y \\
\begin{subarray} { c } { T \neq \emptyset \\
T \cap X = \emptyset } }\end{subarray}} \sum_{\substack{U_{1} \subset T \\
U_{1}=\emptyset}} \Phi\left(\{y\}+U_{1}+Y_{1}\right) \\
& {\left[(\Gamma \Phi)^{-1} * D_{Y-Y_{1}} \Gamma \Phi\right]\left(T-U_{1}\right) \bar{\varrho}_{\Lambda}(X+T)} \\
& =\sum_{\substack{Y_{1} \subset Y \\
U_{1} \cap X=\emptyset \\
U_{1} \neq \emptyset}} \Phi\left(\{y\}+Y_{1}+U_{1}\right) \\
& \cdot \sum_{S \cap\left(X+U_{1}\right)=\emptyset}\left[(\Gamma \Phi)^{-1} * D_{Y-Y_{1}} \Gamma \Phi\right](S) \bar{\varrho}_{\Lambda}\left(X+U_{1}+S\right) .
\end{aligned}
$$


We can use once more the induction hypothesis to obtain finally:

$$
\begin{aligned}
& (\Gamma \Phi)(Y+\{y\}) \varrho_{\Lambda}(X) \\
& \quad=\chi_{\Lambda}(X) \varrho_{\Lambda}(X-Y-\{y\})-\sum_{\substack{T \neq \emptyset \\
T \cap X=\emptyset}}\left[(\Gamma \Phi)^{-1} * D_{\{y\}+Y} \Gamma \Phi\right](T) \varrho_{\Lambda}(X+T) .
\end{aligned}
$$

Corollary. The following set of equations is equivalent to the full set of Eqs. (26):

$$
\Phi\left(x_{1}\right) \bar{\varrho}_{\Lambda}(X)=\chi_{\Lambda}(X) \bar{\varrho}_{\Lambda}\left(X-x_{1}\right)-\sum_{\substack{S \neq \emptyset \\ S \subset \Lambda-X}} \Phi\left(x_{1}+S\right) \bar{\varrho}_{\Lambda}(X+S)
$$

where $x_{1}$ is one of the points of the set $X$, for example the first in lexicographic order.

This last set of equations are the "Kirkwood-Salsburg equations for Polymer Systems". Another subset of equations, analoguous to the Mayer-Montroll equations, is obtained from (26) by taking $Y=X$ :

$(\Gamma \Phi)(X) \bar{\varrho}_{\Lambda}(X)=\chi_{\Lambda}(X)\left[1-\sum_{S \neq \emptyset}\left[(\Gamma \Phi)^{-1} * D_{X} \Gamma \Phi\right](S) \bar{\varrho}_{\Lambda}(X+S)\right]$.

It is expected that this set of equations is also equivalent to the full set (26).

\subsection{Kirkwood-Salsburg Equations for Polymers}

Let $\mathscr{B}_{\xi}$ be the complex Banach space of complex functions $f$ defined on non empty finite subsets of $\mathbb{Z}^{v}$ with the norm

$$
\|f\|_{\xi}=\sup _{X} \frac{|f(X)|}{\xi^{N(X)}}
$$

where $\xi$ is a positive number to be chosen later on.

For any finite $\Lambda, \varrho_{\Lambda}(X)=\chi_{\Lambda}(X) \varrho_{\Lambda}(X)$; moreover for non negative activity

$$
\bar{\varrho}_{\Lambda}(X) \leqq \frac{1}{\Pi \Phi\left(x_{i}\right)} .
$$

Therefore for any finite $\Lambda$, and any non-negative activity $\Phi, \bar{\varrho}_{\Lambda} \in \mathscr{B}_{\xi}$ and satisfies $\left\|\bar{\varrho}_{A}\right\|_{\xi} \leqq 1$ if $\xi \Phi(x)>1$ for all $x$.

We define on $\mathscr{B}_{\xi}$ a linear operator $K_{\Phi}$ as follows: with $f \in \mathscr{B}_{\xi}$

$$
\begin{aligned}
& \left(K_{\Phi} f\right)\left(x_{1}\right)=-\frac{1}{\Phi\left(x_{1}\right)} \sum_{\substack{S \neq \emptyset \\
S \neq x_{1}}} \Phi\left(x_{1}+S\right) f\left(x_{1}+S\right) \\
& \left(K_{\Phi} f\right)(X)=\frac{1}{\Phi\left(x_{1}\right)}\left[f\left(X-x_{1}\right)-\sum_{\substack{S \neq \emptyset \\
S \cap X=\emptyset}} \Phi\left(x_{1}+S\right) f(X+S)\right] \text { if } N(X) \geqq 2 .
\end{aligned}
$$


Lemma 4. For any activity $\Phi$ such that $\sum_{X \ni x} \xi^{N(X)}|\Phi(X)|<\infty$ for some $\xi$ and such that

$$
\min _{x}|\Phi(x)| \neq 0,
$$

the operator $K_{\Phi}$ is a bounded operator on $\mathscr{B}_{\xi}$ and its norm satisfies

$$
\|K\|_{\xi} \leqq \max \frac{1}{|\Phi(x)|} \frac{1}{\xi}\left[1+\sum_{\substack{S \neq x \\ S \neq \emptyset}}|\Phi(x+S)| \xi^{N(x+S)}\right] .
$$

This lemma follows immediately from the definition of $K_{\Phi}$ and $\mathscr{B}_{\xi}$.

Let $\Delta(\xi)$ be the set of complex activities $\Phi$ such that

and

$$
\|\Phi\|_{\xi}=\sup _{x} \sum_{X \ni x}|\Phi(X)| \xi^{N(X)}<\infty
$$

$$
|\Phi(x)|>\sup _{x} \frac{1}{\xi}\left[1+\sum_{\substack{S \neq x \\ S \neq \emptyset}}|\Phi(x+S)| \xi^{N(x+S)}\right]=R(\xi) .
$$

Lemma 5. The operator $K_{\Phi}$ defined on the Banach space $\mathscr{B}_{\xi}$ is norm analytic in $\Phi$ for $\Phi \in \Delta(\xi)$; moreover in this same domain

$$
\left\|K_{\Phi}\right\|_{\xi}<1 .
$$

Proof. A very simple calculation shows that

$$
\left\|K_{\Phi}-K_{\Psi}\right\|_{\xi} \leqq\|\Psi-\Phi\|_{\xi} \quad \text { if } \quad \Phi, \Psi \in \Delta(\xi) .
$$

Analyticity follows from the fact that $K_{\Phi}$ is of the form:

$$
K_{\Phi}=\frac{1}{\Phi\left(x_{1}\right)} \cdot K_{\Phi}^{\prime}
$$

where $\frac{1}{\Phi\left(x_{1}\right)}$ is the operator defined by

$$
\left(\frac{1}{\Phi\left(x_{1}\right)} f\right)(X)=\frac{1}{\Phi\left(x_{1}\right)} f(X)
$$

for all $f \in \mathscr{B}_{\xi}$ and $K_{\Phi}^{\prime}$ is the sum of two operators, one independent of $\Phi$, the other linear in $\Phi$.

Introducing the operator $\chi_{A}$ on $\mathscr{B}_{\xi}$ defined by:

$$
\left(\chi_{\Lambda} f\right)(X)=\chi_{\Lambda}(X) f(X) \text { for all } f \in \mathscr{B}_{\xi}
$$

and denoting by $\alpha$ the vector in $\mathscr{B}_{\xi}$ defined by:

$$
\alpha(X)=\delta_{N(X), 1} \frac{1}{\Phi(x)}
$$


the "Kirkwood-Salsburg equations for polymers" appear as a linear equation on $\mathscr{B}_{\xi}$ of the form:

$$
\bar{\varrho}_{\Lambda}=\chi_{\Lambda} \alpha+\chi_{\Lambda} K_{\Phi} \bar{\varrho}_{\Lambda} .
$$

We can now state the main result of this section:

Theorem 4. Let $\Delta(\xi)$ be defined by (33); then for any $\Phi \in \Delta(\xi)$.

I) For any finite volume $\Lambda$, the "Kirkwood-Salsburg" equation

$$
\bar{\varrho}_{\Lambda}=\chi_{\Lambda} \alpha+\chi_{\Lambda} K_{\Phi} \bar{\varrho}_{\Lambda}
$$

has a unique solution in $\mathscr{B}_{\xi}$, obtained by iteration. This solution is a norm analytic function of $\Phi$ in $\Delta(\xi)$ and satisfies

$$
\left\|\bar{\varrho}_{\Lambda}\right\|_{\xi} \leqq\left\{1-\max _{x} \frac{1}{|\Phi(x)| \xi}\left[1+\sum_{\substack{S \neq \emptyset \\ S \notin x}}|\Phi(x+S)| \xi^{N(S)+1}\right]\right\}^{-1}
$$

II) For any finite volume $\Lambda, Q_{\Lambda}[\Phi] \neq 0$, and the solution of the Kirkwood-Salsburg equation coincide with the definition (18).

III) The infinite volume equation

$$
\bar{\varrho}=\alpha+K_{\Phi} \bar{Q}
$$

has a unique solution in $\mathscr{B}_{\xi}$, which is also analytic in $\Delta(\xi)$. Moreover if the activity is invariant under a certain subgroup $T$ of translation then $\bar{\varrho}$ is also invariant under $T$.

IV) If the activity is invariant under a certain subgroup $T$ of translations such that the quotient group $\mathbb{Z}^{v} / T$ is finite (i.e. $T$ is generated by $v$ elementary translations)

or if the activity has finite range,

then there exists a positive, decreasing function $\varepsilon(\lambda)$ such that

$$
\lim _{\lambda \rightarrow \infty} \varepsilon(\lambda)=0
$$

and

$$
\left|\bar{\varrho}_{\Lambda}(X)-\bar{\varrho}(X)\right| \leqq \xi^{N(X)} \varepsilon(\lambda)
$$

where $\lambda$ is the minimum distance from $x_{i} \in X$ to the boundary of $\Lambda$.

$\mathrm{V})$ if the activity is invariant under a subgroup $T$ of translation (such that the order of $\mathbb{Z}^{v} / T$ is finite), then:

$$
\bar{\varrho}(X)=\lim _{\Lambda \rightarrow \infty} \frac{N\left(\mathbb{Z}^{v} / T\right)}{N(\Lambda)} \sum_{\tau \in T} \bar{\varrho}_{\Lambda}(\tau X)
$$

where $N\left(\mathbb{Z}^{v} / T\right)$ is the order of the quotient group $\mathbb{Z}^{v} / T$ and $\Lambda$ tends to infinity in the sense of Van Hove. Moreover the convergence is uniform with respect to $\Phi(x)$ on every compact subset contained in $\Delta(\xi)$. 
Proof. Part I follows from Lemma 1 and 2 and the fact that $\chi_{\Lambda} \alpha$ is a vector which is norm analytic for $\Phi \in \Delta(\xi)$.

Part II is a consequence of the fact that $\varrho_{\Lambda}(x)$ is analytic in $\Phi(x)$ and $\bar{\varrho}_{\Lambda}(x)=\frac{d}{d \Phi(x)} \log Q_{\Lambda}$.

Part III is obtained from Lemma 4 and 5 and the fact that if $\Phi(X)$ is invariant under a subgroup $T$ then $\alpha$ and $K_{\Phi}$ are invariant under $T$.

Using the same argument as Ruelle [9], part IV will be proved if we can show that for $\Lambda \subset \Lambda^{\prime} \subset \Lambda^{\prime \prime}$

$$
\left\|\chi_{\Lambda} K_{\Phi} \chi_{\Lambda^{\prime \prime}}-\chi_{\Lambda} K_{\Phi} \chi_{\Lambda^{\prime}}\right\|_{\xi} \leqq \eta(\delta)
$$

where $\delta$ is the minimum distance from $\Lambda$ to the boundary of $\Lambda^{\prime}$ and $\eta(\delta)$ satisfies $\lim _{\delta \rightarrow \infty} \eta(\delta)=0$.

This inequality follows from:

$$
\begin{aligned}
& \left|\chi_{\Lambda}(X)\left(K_{\Phi} \chi_{\Lambda^{\prime \prime}} f\right)(X)-\chi_{\Lambda}(X)\left(K_{\Phi} \chi_{\Lambda^{\prime}} f\right)(X)\right| \\
\leqq & \frac{1}{\left|\Phi\left(x_{1}\right)\right|} \sum_{\substack{S \neq \emptyset \\
S \neq x_{1}}}\left|\Phi\left(x_{1}+S\right)\right||f(X+S)|\left(1-\chi_{\Lambda^{\prime}}(S)\right)
\end{aligned}
$$

and the fact that $\Phi \in \Delta(\xi)$ which yields

where

$$
\left\|\chi_{\Lambda} K_{\Phi} \chi_{\Lambda^{\prime \prime}}-\chi_{\Lambda} K_{\Phi} \chi_{\Lambda^{\prime}}\right\|_{\xi} \leqq \sup _{x_{1}} \sum_{\substack{S \neq \emptyset \\ S \neq x_{1} \\ S \nsubseteq \Lambda^{\prime}}}\left|\Phi\left(x_{1}+S\right)\right| \xi^{N(S)+1} \leqq \eta(\delta)
$$

$$
\eta(\delta)=\sup _{x_{1}} \sum_{\substack{S \neq \emptyset \\ S \neq x_{1} \\ S \nmid B_{\delta}\left(x_{1}\right)}}\left|\Phi\left(x_{1}+S\right)\right| \xi^{N(S)+1}
$$

and $B_{\delta}\left(x_{1}\right)$ denotes the ball of radius $\delta$ centered at $x_{1}$.

Moreover if the activity has finite range $\Delta, \eta(\delta)=0$ if $\delta>\delta_{0}$ where $\delta_{0}$ is the diameter of the range; on the other hand if the activities are invariant under $T$

and since

$$
\eta(\delta)=\sup _{\tau \in \mathbb{Z}^{v} / T} \sum_{\substack{S \neq \emptyset \\ S \neq \tau x \\ S \notin B_{\delta}(\tau x)}}|\Phi(\tau x+S)| \xi^{N(S)+1}
$$

$$
\sup _{x} \sum_{S \ngtr x}|\Phi(x+S)| \xi^{N(S)+1}<\infty
$$

then $\lim _{\delta \rightarrow \infty} \eta(\delta)=0$ since the order of $\mathbb{Z}^{\nu} / T$ is finite.

Finally the proof of part $V$ is identical with the one given in reference [8]; moreover the limit is uniform on every compact set contained in 
$\Delta(\xi)$ since $\left|\varrho_{\Lambda}(X)\right|$ has a bound independent of $X$ and which can be chosen independent of $\Phi(x)$ if $\Phi$ belongs to a compact set in $\Delta(\xi)$.

Remark. Part IV of the last theorem can be moreover extended to all $\Phi \in \Delta(\xi)$ which are the strong limit (in the norm $\|\cdot\|_{\xi}$ ) of $\Phi_{\delta}$

where

$$
\Phi_{\delta}=\left\{\begin{array}{l}
\Phi(X) \text { if }\left|x_{i}-x_{j}\right|<\delta \text { for all } i, j \\
0 \text { otherwise }
\end{array}\right.
$$

In the study of phase transition one is usually interested in the domain of analyticity of $\varrho$ as function of the monomer activity for fixed value of the activity of the other polymers. In this case the previous theorem reads:

Theorem 5. The solutions $\bar{\varrho}_{A}$ and $\bar{\varrho}$ of the Kirkwood-Salsburg equations are analytic functions of the monomer activity $\Phi(x)$ in the domain:

$$
|\Phi(x)|>\max _{x} \frac{1}{\xi_{0}}\left[1+\sum_{\substack{S \neq \emptyset \\ S \neq x}}|\Phi(x+S)| \xi_{0}^{N(S)+1}\right]=R\left(\xi_{0}\right)
$$

where $\xi_{0}$ is the value of $\xi$ such that $R\left(\xi_{0}\right)$ is minimum.

In particular if the activity is translationally invariant $\xi_{0}$ is the solution of

$$
1=\sum_{S \ni 0}(N(S)-1)|\Phi(S)| \xi_{0}^{N(S)} .
$$

Remark. In all those types of results, one would be interested to know how good is the domain of analyticity thus obtained; one can show that this is in particular the best radius of convergence for systems consisting of monomers and n-mers ( $n$ fixed); indeed there exists special types of interactions (Van der Waals) for which the radius obtained in Theorem 4 is the exact radius of convergence.

\subsection{Mayer Series and Virial Expansion}

From Theorem 4, part V, we have that if $\Lambda \rightarrow \infty$ in the sense of Van Hove, and if the activity is invariant under translation, then

$$
\lim _{\Lambda \rightarrow \infty} \frac{1}{N(\Lambda)} \sum_{x} \bar{\varrho}_{\Lambda}(x)=\bar{\varrho}^{(1)}=\frac{\varrho^{(1)}}{z}
$$

uniformly with respect to $z$ on every compact subset contained in the exterior of the circle of radius $R\left(\xi_{0}\right)$.

Moreover since

$$
\frac{1}{N(\Lambda)} \sum_{x} \bar{\varrho}_{\Lambda}(x)-\frac{1}{z}=\frac{d}{d z}\left(\beta p_{\Lambda}-\log z\right)
$$


and

$$
\lim _{z \rightarrow \infty}\left(\beta p_{\Lambda}-\log z\right)=0
$$

we have

$$
\begin{gathered}
\beta p_{\Lambda}=\log z-\int_{z}^{\infty} d z\left(\frac{1}{N(\Lambda)} \sum_{x} \bar{\varrho}_{\Lambda}(x)-\frac{1}{z}\right) \\
\beta p=\lim _{\Lambda \rightarrow \infty} \beta p_{\Lambda}=\log z+\int_{z}^{\infty} d z \frac{1-\varrho^{(1)}}{z} .
\end{gathered}
$$

In conclusion we have just proved:

Theorem 6. If $\Lambda \rightarrow \infty$ in the sense of Van Hove, then the following limit exists

$$
\begin{aligned}
& \lim _{\Lambda \rightarrow \infty} \frac{1}{N(\Lambda)} \log Q_{\Lambda}=\beta p, \\
& \lim _{\Lambda \rightarrow \infty} z \frac{d}{d z} \frac{1}{N(\Lambda)} \log Q_{\Lambda}=\varrho^{(1)}
\end{aligned}
$$

when the activity is translationally invariant and $|z|>R(\xi)$.

These limits continue analytically to $|z|>R\left(\xi_{0}\right)$ the physical pressure and density defined for $z>0$. In particular the Mayer series

$$
\begin{gathered}
\beta p=\log z+\sum_{n=1}^{\infty} b_{n} z^{-n}, \\
\varrho^{(1)}=1-\sum_{n=1}^{\infty} n b_{n} z^{-n}
\end{gathered}
$$

has a radius of convergence of at least $R\left(\xi_{0}\right)^{-1}$ in $z^{-1}$.

Instead of the Mayer series (44) one would like to obtain an expansion of the pressure $p$ in powers of the monomer density $\varrho^{(1)}$, that is the Virial expansion; in our case $\varrho^{(1)}$ being analytic around $z^{-1}=0$, we shall look for an expansion in powers of $\left(1-\varrho^{(1)}\right)$.

From

follows that

$$
\bar{\varrho}(x)=\sum_{k=0}^{\infty}\left(K^{k} \alpha\right)(x)
$$

$$
1-\varrho^{(1)}=-z \sum_{k=1}^{\infty}\left(K^{k} \alpha\right)(x)
$$

and therefore the first non-zero coefficient of the Mayer series (45) is $b_{p}, p \geqq 2$, where $p$ is the smallest number $>1$ such that $\Phi(X) \neq 0$ for some $N(X)=p$; moreover in this case

$$
p b_{p}=\sum_{\substack{X \ni 0 \\ N(X)=p}} \Phi(X) .
$$


In particular $b_{1}=0$ and in general $p$ and $z$ will not be analytic in $\varrho^{(1)}$ at $\varrho^{(1)}=1$; however we shall now derive the virial expansion for the special case of a system made up only of monomers and $n p$-mers ( $p$ fixed, $n=1,2, \ldots)$. For this special case we have:

that is

$$
1-\varrho^{(1)}=-z \sum_{l=1}^{\infty}\left(K^{p l} \alpha\right)(x)
$$

$$
\begin{aligned}
1-\varrho^{(1)} & =\sum_{l=1}^{\infty}(p l) b_{p l} z^{-p l}, \\
\beta p-\log z & =\sum_{l=1}^{\infty} b_{p l} z^{-p l} .
\end{aligned}
$$

Since by assumption $p b_{p} \neq 0$, we may invert (48) in a neighborhood of $z^{-1}=0$ and we obtain the virial expansion

where

$$
\beta p-\log z=\sum_{n=1}^{\infty} c_{n}\left(1-\varrho^{(1)}\right)^{n}
$$

$$
c_{n}=-\frac{1}{2 i \pi} \int_{c} \frac{d z}{z} \frac{1}{n\left(1-\varrho^{(1)}\right)^{n-1}}
$$

if $c$ is a circle of radius larger than $R\left(\xi_{0}\right)$.

From (47) follows that:

$$
\left|1-\varrho^{(1)}+z\left(K^{p} \alpha\right)(x)\right|=\left|1-\varrho^{(1)}-p b_{p}\right| \leqq \frac{\|K\|_{\xi}^{2 p}}{1-\|K\|_{\xi}^{p}}
$$

therefore for $|z|>R\left(\xi_{0}\right)=R\left(R\left(\xi_{0}\right)\right.$ defined by $\left.(42)\right)$

$$
\left|1-\varrho^{(1)}\right| \geqq \frac{\left|p b_{p}\right|}{|z|^{p}}-\frac{R^{2 p}}{z^{2 p}-z^{p} R^{p}} .
$$

In conclusion we obtain:

$$
\left|1-\varrho^{(1)}\right| \geqq \frac{\left|p b_{p}\right|}{R^{p}}+2\left[1-\left(1+\frac{\mid p b_{p} p}{R^{p}}\right)^{\frac{1}{2}}\right]
$$

on the circle $|z|^{-p}=R^{-p}-\left[R^{p}\left(\left|p b_{p}\right|+R^{p}\right)\right]^{-\frac{1}{2}}$.

We thus obtain the following theorem:

Theorem 7. The radius of convergence of the virial expansion

is at least

$$
\beta p-\log z=\sum_{n=1}^{\infty} c_{n}\left(1-\varrho^{(1)}\right)^{n}
$$

$$
\left|1-\varrho^{(1)}\right|=\frac{\left|p b_{p}\right|}{R^{p}}+2\left[1-\left(1+\frac{\left|p b_{p}\right|}{R^{p}}\right)^{\frac{1}{2}}\right]
$$


where

$$
p b_{p}=\sum_{\substack{X \ni 0 \\ N(X)=p}} \Phi(X)
$$

and $R=R\left(\xi_{0}\right)$ was defined in (42).

Finally we shall remark that because of the scaling property:

$$
\begin{aligned}
& \beta p[\Phi]=\beta p\left[z^{-N} \Phi\right], \\
& \varrho^{(1)}[\Phi]=\varrho^{(1)}\left[z^{-N} \Phi\right]
\end{aligned}
$$

we can always choose $z=1$ and thus obtain the usual form of the virial expansion:

$$
\beta p=\sum_{n=1}^{\infty} c_{n}\left(1-\varrho^{(1)}\right)^{n} .
$$

To conclude this section we mention the following generalization of Theorem 7:

Theorem 8. For any polymer system the virial expansion

$$
\beta p-\log z=\sum_{n=1}^{\infty} c_{n}\left(1-\varrho^{(1)}\right)^{\frac{n}{m}}
$$

where $m$ is the smallest $r$ such that $\Phi\left(x_{1}, \ldots, x_{r}\right) \neq 0$ converges in a neighbourhood of $\varrho^{(1)}=1$.

\section{Monomer-Dimer Systems}

For the special case of monomer-dimer systems the domain of analycity of the pressure and the correlation functions, as functions of the monomer activity (obtained in $\S 4$ ) can be extended by studying the zeros of the partition function.

\subsection{Zeros of the Partition Function and Extension of the Domain of $p$ and $\bar{\varrho}$}

Theorem 9. The partition function $Q_{\Lambda}[\Phi]$ is different from zero for any complex activities such that $\operatorname{Re} \Phi(x)>0 \forall x \in \Lambda$ or $\operatorname{Re} \Phi(x)<0 \forall x \in \Lambda$ and $\Phi(x, y) \geqq 0$ for all $x, y \in \Lambda$.

We shall prove this theorem by induction using the recurrence formula (14) which gives:

$$
Q_{\Lambda}[\Phi]=\Phi\left(x_{i}\right) Q_{\Lambda-x_{i}}[\Phi]+\sum_{\substack{x_{j} \in \Lambda \\ j \neq i}} \Phi\left(x_{i}, x_{j}\right) Q_{\Lambda-x_{\imath}-x_{j}}[\Phi]
$$


1. Let us first consider the case: $\operatorname{Re} \Phi(x)>0, \Phi(x, y) \geqq 0$.

i) $Q_{\{x\}}[\Phi]=\Phi(x)$,

$$
Q_{\left\{x_{1}, x_{2}\right\}}[\Phi]=\Phi\left(x_{1}, x_{2}\right)+\Phi\left(x_{1}\right) \Phi\left(x_{2}\right)
$$

therefore

and

$$
Q_{\{x\}} \neq 0 \quad \text { and } \quad Q_{\left\{x_{1}, x_{2}\right\}} \neq 0
$$

$$
\operatorname{Re}\left[Q_{\left\{x_{1}, x_{2}\right\}}[\Phi] Q_{\left\{x_{1}\right\}}^{*}[\Phi]\right]=\operatorname{Re} \Phi^{*}\left(x_{1}\right) \Phi\left(x_{1}, x_{2}\right)+\operatorname{Re} \Phi\left(x_{2}\right)\left|\Phi\left(x_{1}\right)\right|^{2}>0 .
$$

ii) Assuming $Q_{X}[\Phi] \neq 0$ and

$$
\operatorname{Re}\left[Q_{X}[\Phi] Q_{X-x_{t}}^{*}[\Phi]\right]>0
$$

for all $X$ such that $N(X) \leqq n-1$ and for all $x_{i} \in X$ let us show the same holds for all $X$ such that $N(X)=n$; using (51) we have:

$$
\begin{gathered}
Q_{X}[\Phi] Q_{X-x_{i}}^{*}[\Phi]=\left[\Phi\left(x_{i}\right) Q_{X-x_{i}}+\sum_{j \neq i} \Phi\left(x_{i}, x_{j}\right) Q_{X-x_{i}-x_{j}}\right] Q_{X-x_{i}}^{*} \\
=\Phi\left(x_{i}\right)\left|Q_{X-x_{i}}\right|^{2}+\sum_{j \neq i} \Phi\left(x_{i}, x_{j}\right) Q_{X-x_{i}}^{*} Q_{X-x_{i}-x_{j}} .
\end{gathered}
$$

Therefore

$$
\operatorname{Re}\left(Q_{X}[\Phi] Q_{X-x_{\imath}}^{*}[\Phi]\right)>0 \text { for all } x_{i} \in X
$$
and $Q_{X}[\Phi] \neq 0$.

2. Using the scaling property $\left|Q_{\Lambda}[\Phi]\right|=\left|Q_{\Lambda}\left[(-1)^{N} \Phi\right]\right|$ the theorem is also true for the case $\operatorname{Re} \Phi(x)<0$ which concludes the proof. This theorem enables us to extend the domain of analyticity of the pressure and the correlation functions, and we have:

Theorem 10. For monomer-dimer systems such that the activity is stable, translationally invariant, and satisfies $\Phi(x, y) \geqq 0$, the following limit exists

$$
\begin{aligned}
& \lim _{\Lambda \rightarrow \infty} \frac{1}{N(\Lambda)} \log Q_{\Lambda}=\beta p, \\
& \lim _{\Lambda \rightarrow \infty} \bar{\varrho}_{\Lambda}(X)=\bar{\varrho}(X)
\end{aligned}
$$

when $\Lambda \rightarrow \infty$ in the sense of Van Hove and $z \notin\left[-2 i W^{\frac{1}{2}}, 2 i W^{\frac{1}{2}}\right]$ where $W=\sum_{y \neq 0} \Phi(0, y)$. Moreover in this domain $p$ and $\varrho(X)$ are the analytic continuation of the physical pressure and reduced correlation function (defined for $z \geqq 0$ ). 
Proof. The previous theorems imply that $p_{\Lambda}=\frac{1}{N(\Lambda)} \log Q_{\Lambda}$ and $\bar{\varrho}_{\Lambda}(X)$ are analytic function of $z$ in the plane cut by $\left[-2 i W^{\frac{1}{2}}, 2 i W^{\frac{1}{2}}\right]$; moreover $p_{A}$ and $\bar{\varrho}_{A}(X)$ are uniformly bounded on any compact subset of the $z$-plane cut by this segment: indeed from the proof of Lemma 1 follows that

$$
\frac{1}{N(\Lambda)} \log \left|Q_{\Lambda}\right| \leqq|z|+\frac{1}{2} W
$$

while relations (51) and (52) yields for $\operatorname{Re} z>0$

$$
\begin{gathered}
\left|Q_{\Lambda} Q_{\Lambda-x}^{*}\right| \geqq \operatorname{Re} Q_{\Lambda} Q_{\Lambda-x}^{*} \geqq|\operatorname{Re} z|\left|Q_{\Lambda-x}\right|^{2}, \\
\left|Q_{\Lambda}\right| \geqq|\operatorname{Re} z|\left|Q_{\Lambda-x}\right| \geqq|\operatorname{Re} z|^{N(X)}\left|Q_{\Lambda-x}\right|
\end{gathered}
$$

and using the scalling relation $\left|Q_{\Lambda}[\Phi]\right|=\left|Q_{\Lambda}\left[(-1)^{N} \Phi\right]\right|$ this inequality remains valid for $\operatorname{Re} z<0$.

Therefore $\left|\varrho_{\Lambda}(X)\right| \leqq|\operatorname{Re} z|^{-N(X)}$.

This result together with Theorem 4 shows that $\bar{\varrho}_{A}(X)$ is uniformly bounded on any compact subset of the $z$-plane cut by $[-2 i \sqrt{W}, 2 i \sqrt{W}]$.

Finally Theorem 4 and 6 imply that $\bar{p}_{A} \rightarrow p$ and $\bar{\varrho}_{A}(X) \rightarrow \bar{\varrho}(X)$ for $|z|>2 W^{\frac{1}{2}}$; we can therefore apply Vitali's theorem to conclude that $p_{A}$ and $\bar{\varrho}_{\Lambda}(X)$ converge uniformly on compact subsets to an analytic function on the $z$-plane cut by the segment $\left[-2 i W^{\frac{1}{2}}, 2 i W^{\frac{1}{2}}\right]$.

\section{Conclusion}

No phase transition can occur in monomer-dimer systems if the activity is stable, translationally invariant and satisfies $\Phi(x) \neq 0 \Phi(x, y) \geqq 0$.

Finally Theorem 9 and 10 enable us to obtain other properties concerning the functions $\varrho^{(1)}$ and $p$.

Theorem 11. The monomer density

$$
\varrho^{(1)}(z)=\sum_{j=0}^{\infty} a_{j}\left(-z^{-2}\right)^{j}=\sum_{j=0}^{\infty} a_{j}(-\zeta)^{j}
$$

is a series of Stieltjes in $\zeta=z^{-2}$ and therefore

$$
\varrho^{(1)}(z)=\int_{0}^{4 W} \frac{d f(y)}{1+y z^{-2}}
$$

where $f(y)$ is a bounded, non decreasing function. 
Proof. Let $u=\frac{1-z}{1+z}$; Theorem 9 implies that $\varrho^{(1)}$, as function of $u$, is analytic in the circle $|u|<1$; moreover from (52) follows that $\operatorname{Re} \varrho^{(1)}>0$ for $|u|<1$.

From those properties follows that [12] the function

$$
F(w)=\frac{1}{\sqrt{1+w}} \bar{\varrho}^{(1)}\left((1+w)^{-\frac{1}{2}}\right)
$$

is a series of Stieltjes in $w$; therefore $\varrho^{(1)}(z)=z \bar{\varrho}^{(1)}(z)$ is a series of Stieltjes in $\left(z^{-2}-1\right)$ and also a series of Stieltjes in $\zeta=z^{-2}$.

Theorem 12. Let $[N, M](\zeta)$ denote the Pade approximant for the series of Stieltjes of $\varrho^{(1)}$; for $z$ real, non negative, the monomer density satisfies:

$$
\begin{gathered}
{[N, N]\left(\zeta=z^{-2}\right) \geqq \varrho^{(1)}(z) \geqq[N, N-1]\left(\zeta=z^{-2}\right)} \\
-\frac{d}{d \zeta}[N, N]\left(\zeta=z^{-2}\right) \leqq \frac{1}{2} z^{3} \frac{d \varrho^{(1)}(z)}{d z} \leqq \frac{d}{d \zeta}[N, N-1]\left(\zeta=z^{-2}\right)
\end{gathered}
$$

and the pressure is an increasing continuous function of the monomer density.

Proof. The first part of this theorem is a direct consequence [13] of Theorem 11. The last part follows from

$$
\frac{d p}{d \varrho^{(1)}}=\frac{\varrho^{(1)}}{z \frac{d \varrho^{(1)}}{d z}}
$$

and the evaluation of the first Padé approximants:

$$
\begin{aligned}
{[1,0](\zeta) } & =\frac{1}{1+W \zeta} \quad[1,1](\zeta)=\frac{1+a \zeta}{1+b \zeta} \\
a & =W-W^{-1} \sum_{s} \Phi(0, s)^{2} \\
b & =W+a .
\end{aligned}
$$

\section{The Association Problem}

In this section we want to generalize an argument given by Green and Hurst [1] to show that the study of any classical lattice system can be reduced to the study of a polymer system on the same lattice. 


\subsection{Lattice Gas}

We consider a classical system of particles on $\mathbb{Z}^{v}$ such that at each lattice point there can be 0 or 1 particle; the system is described by the Hamiltonian:

$$
H(X)=-\mu N(X)+U(X) \quad X \subset \mathbb{Z}^{v}
$$

where the potential energy $U(X)$ is assumed to be translationnally invariant and $U(X)=0$ if $N(X)<2$; with $\mu$ the chemical potential, the activity $z$ is given by $z=e^{\beta \mu}$.

For any finite volume $\Lambda$, the partition function $Z_{A}$ and the correlation functions $\varrho_{A}(X)$ are defined as:

$$
\begin{aligned}
Z_{\Lambda}=\sum_{Y \subset A} e^{-\beta H(Y)} & =\sum_{Y \subset A} z^{N(Y)} e^{-\beta U(Y)} \\
\varrho_{\Lambda}(X)=Z_{\Lambda}^{-1} \sum_{Y \subset \Lambda-X} e^{-\beta H(X+Y)} & =Z_{\Lambda}^{-1} \sum_{Y \subset A-X} z^{N(X+Y)} e^{-\beta U(X+Y)} .
\end{aligned}
$$

Finally we introduce the Ursell Functions $\Psi(X)$ defined by:

$$
(\Gamma \Psi)(X)=e^{-\beta U(X)} ; \quad \Psi(x)=1 .
$$

Lemma 6. i) The partition function $Z_{A}$ for the lattice gas described by the activity $z$ and the Ursell function $\Psi(X)$ is up to a factor $z^{N(A)}$ identical with the partition function of a polymer system described by the activity:

$$
\begin{array}{cll}
\Phi(x)=\frac{1}{z}+1 & \text { if } & N(X)=1, \\
\Phi(X)=\Psi(X) & \text { if } & N(X) \geqq 2
\end{array}
$$$$
\text { that is: } \quad Z_{\Lambda,(z, \Psi)}^{\text {Lattice }}=z^{N(\Lambda)} Q_{\Lambda,\left(\frac{\delta}{z}+\Psi\right)}^{\text {Polymer }} \quad \text { where } \quad \delta(X)=\delta_{N(X), 1} .
$$

ii) The correlation functions $\varrho_{A}^{\mathrm{Lattice}}(X)$ of the lattice gas are linear combinations of the correlation functions $\bar{\varrho}_{A}^{\text {Polymer }}(X)$ of the polymer system described by the activity $\Phi(X)$ given in part (i):

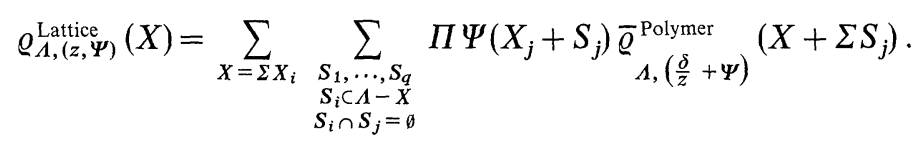

Proof. i) $Z_{\Lambda,(z, \Psi)}^{\mathrm{Lattice}}=\sum_{Y \subset A} z^{N(Y)}(\Gamma \Psi)(Y)=\Gamma\left(\delta+z^{N} \Psi\right)(\Lambda)$ therefore:

$$
Z_{\Lambda,(z, \Psi)}^{\text {Lattice }}=Q_{\Lambda,\left(\delta+z^{N} \Psi\right)}^{\text {Polymer }}=z^{N(\Lambda)} Q_{\Lambda,\left(\frac{\delta}{z}+\Psi\right)}^{\text {Polymer }}
$$


ii) $\varrho_{\Lambda,\left(z, \Psi^{\prime}\right)}^{\text {Lattice }}=\frac{1}{Z_{\Lambda}} \sum_{Y \subset A-X}\left[D_{X} \Gamma\left(z^{N} \Psi\right)\right](Y)$

$$
\begin{aligned}
& =\frac{1}{Z_{A}} \sum_{Y \subset A-X} \sum_{X=\Sigma X_{j}} \sum_{\substack{S_{1} \ldots S_{q} \\
S_{j} \subset Y \\
S_{\imath} \cap S_{j}=\emptyset}} \prod_{j}\left(z^{N} \Psi\right)\left(X_{j}+S_{j}\right)\left[\Gamma\left(z^{N} \Psi\right)\right]\left(Y-\Sigma S_{j}\right) \\
& =\sum_{X=\Sigma X_{j}} \sum_{\substack{S_{1} \ldots S_{q} \\
S_{j} \subset A-X \\
S_{i} \cap S_{j}=\emptyset}} \prod_{j}\left(z^{N} \Psi\right)\left(X_{j}+S_{j}\right) \cdot \sum_{T \subset A-X-\Sigma S_{j}} \frac{\Gamma\left(z^{N} \Psi\right)(T)}{Z_{\Lambda}} .
\end{aligned}
$$

But

$\frac{1}{Z_{\Lambda}} \sum_{T \subset \Lambda-X-S} \Gamma\left(z^{N} \Psi\right)(T)=\frac{1}{Z_{\Lambda}} \Gamma\left(\delta+z^{N} \Psi\right)(\Lambda-X-S)=\bar{\varrho}_{\Lambda,\left(\delta+z^{N} \Psi\right)}(X+S)$.

Using the scaling property (22) we arrive at the relation (54) which ends the proof.

Theorem 13. Consider a classical lattice gas described by the Hamiltonian $H(X)=-\mu N(X)+U(X)$ where the potential energy is invariant under translation.

1. If the Ursell functions $\Psi(X)=\left(\Gamma^{-1} e^{-\beta U}\right)(X)$ satisfy the conditions

i) $\Psi(X)>0$,

ii) $\sum_{X \ni 0} \xi^{N(X)} \frac{\Psi(X)}{N(X)}<\infty$ for some $\xi>0$

then the following limit exists and is finite:

$$
\lim _{\Lambda \rightarrow \infty} \frac{1}{N(\Lambda)} \log Z_{\Lambda}^{\text {Lattice }}=p
$$

2. If there exists a real number $\xi$ such that

$$
\sum_{\substack{X \not 0 \\ X \neq \emptyset}}|\Psi(0, X)| \xi^{N(X)}<\infty
$$

then in the domain

$$
\left|\frac{1+z}{z}\right|<\xi^{-1}\left\{1+\sum_{\substack{X \neq 0 \\ X \neq \emptyset}}|\Psi(0, X)| \xi^{N(X)+1}\right\}
$$


the following limit exists

$$
\lim _{\Lambda \rightarrow \infty} \varrho_{\Lambda}^{\text {Lattice }}(X)=\varrho^{\text {Lattice }}(X)
$$

and is analytic in $z$.

Proof. Part 1 follows from Lemma (6) and Theorem (6).

To show the second part, we use Theorem (5) which implies that for

$$
\left|\frac{1}{z}+1\right| \geqq \frac{1}{\xi}\left\{1+\sum_{\substack{S \neq \emptyset \\ S \neq 0}}|\Psi(0, S)| \xi^{N(S)+1}\right\}
$$

$\varrho_{\Lambda, \frac{\delta}{z}+\Psi}^{\text {Polymer }}(X)$ and $\varrho_{\frac{\delta}{z}+\Psi}^{\text {Polymer }}(X)$ are analytic in $z$; moreover for any $(z, \Psi)$ in this domain

$$
\left|\varrho_{\Lambda,(z, \Psi)}^{\text {Latice }}(X)\right| \leqq \sum_{X=\Sigma X_{i}} \prod_{j} \sum_{S_{j}}\left|\Psi\left(X_{j}+S_{j}\right)\right| \xi^{N\left(X_{j}+S_{j}\right)} \cdot\left\|\varrho_{\Lambda,\left(\frac{\delta}{z}+\Psi\right)}^{\text {Polymer }}\right\|_{\xi}
$$

which yields

$$
\left|\varrho_{\Lambda,(z, \Psi)}^{\text {Lattice }}(X)\right| \leqq \sum_{q=1}^{N(X)} q^{N(X)}\left[\sum_{S} \Psi(0, S) \xi^{N(S)+1}\right]^{q}\left\|\varrho_{\Lambda,\left(\frac{\delta}{z}+\Psi\right)}^{\text {Polymer }}\right\|_{\xi}
$$

Therefore the series defining $\varrho_{\Lambda,(z, \Psi)}^{\text {Lattice }}(X)$ is absolutely convergent; the rest follows from the uniform convergence of $\bar{\varrho}_{\Lambda}^{\text {Polymer }}$ to $\bar{\varrho}^{\text {Polymer }}$ when $\Lambda \rightarrow \infty$.

\subsection{General Lattice System}

We recall that with each point $x$ of a finite set $\Lambda \subset \mathbb{Z}^{v}$ is associated a given subsystem whose states are numbered by $n_{x} \in\{0,1, \ldots, M\}$.

The configuration of this system $\Lambda$ are caracterised by the $N$-tuple:

$$
n_{\Lambda}=\left(n_{x_{1}}, \ldots, n_{x_{N}}\right) \quad N=N(\Lambda)
$$

The correlation functions are defined by:

$$
\varrho_{\Lambda}^{\text {Lattice }}\left(n_{X}\right)=\frac{1}{Q_{\Lambda}} \sum_{n_{\Lambda-X}} e^{-\beta H\left(n_{\Lambda}\right)}
$$


where $Q_{\Lambda}^{\mathrm{Lattice}}=\sum_{n_{\Lambda}} e^{-\beta H\left(n_{A}\right)} \quad$ is the partition function and

$$
\begin{aligned}
H\left(n_{\Lambda}\right) & =U\left(n_{\Lambda}\right)-\mu\left(n_{A}\right) \\
\mu\left(n_{\Lambda}\right) & =\sum_{x} \mu\left(n_{x}\right) ; \\
\mu\left(n_{x}\right) & =\text { chemical potential of the state } n_{x} \\
U\left(n_{A}\right) & =\text { potential energy corresponding to the configuration } n_{\Lambda} .
\end{aligned}
$$

In this case the Ursell Functions $\Psi\left(n_{X}\right)$ can again be introduced by means of a $*$-exponential where the $*$-product is defined by:

and we have:

$$
\left(f_{1} * f_{2}\right)\left(n_{X}\right)=\sum_{Y \subset X} f_{1}\left(n_{Y}\right) f_{2}\left(n_{X-Y}\right)
$$

$$
e^{-\beta U\left(n_{X}\right)}=(\Gamma \Psi)\left(n_{X}\right) \quad \Psi\left(n_{x}\right)=1 .
$$

We give without proof the following result:

Theorem 14. i) $Q_{\Lambda}^{\text {Lattice }}=Q_{\Lambda}^{\text {Polymer }}[\Phi]$

where

ii)

$$
\Phi(X)=\sum_{n_{X}} e^{\beta \mu\left(n_{X}\right)} \Psi\left(n_{X}\right) .
$$

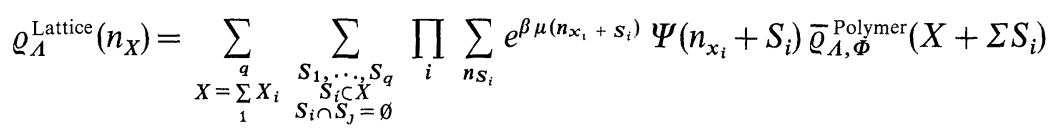

where $\Phi(X)$ is given by (55).

In conclusion from the existence and analyticity property of the pressure and correlation functions for polymer systems we can deduce analoguous results for the pressure and correlation functions of general lattice systems.

\section{References}

1. Green, H. S., Leipnik, R.: Rev. Mod. Phys. 32, 129 (1960).

Kasteleyn, P. W.: Physica 27, 1209 (1961). - J. Math. Phys. 4, 287 (1963); - Graph Theory and Theoretical Physics. New York: Academic Press 1967.

2. Levelt, J. M. H., Cohen, E. G. D.: Studies in Statistical Mechanics, Vol. II. Amsterdam: North Holland Publ. 1964.

3. Ruelle, D.: Statistical Mechanics, Section 2.4. New York: Benjamin 1969.

4. Heilmann, O.J., Lieb, E. H.: Phys. Rev. Letters 24, 1412 (1970).

5. Ruelle, D.: (Ref. [3]) Section 4.4.

6. - (Ref. [3]) Exercice 3D, p. 64.

7. - (Ref. [3]) Section 7.3.

8. Gallavotti, G., Miracle-Sole, S.: Commun. math. Phys. 7, 274 (1968). 
9. Ruelle, D.: (Ref. [3]) Section 4.2.

10. Green,H.S., Hurst, C.A.: Order-Disorder Phenomena Monograph in Statistical Physics, Vol. 5. New York: Interscience 1964.

11. Kunz, H.: Phys. Letters 32 A, 311 (1970).

12. Baker,G., Gammel,J.: The Padé Approximant in Theoretical Physics, Theorem 10, p. 18-19. New York: Academic Press 1970.

13. - - The Padé Approximant in Theoretical Physics, Theorem 7, p. 11. New York: Academic Press 1970.

Dr. C. Gruber

H. Kunz

Ecole Polytechnique Fédérale

Département de Physique Théorique

CH 1006-Lausanne, Schweiz 